\section{Histoire Épistémologie Langage}

43-2 | 2021

Linguistique psychologique et sémiotique : le contexte allemand et son influence

\title{
Larcher, Pierre. 2020. Sur le Coran. Nouvelles approches linguistiques
}

Limoges : Lambert-Lucas. 235 p.

\section{Julien Sibileau}

\section{OpenEdition \\ Journals}

Édition électronique

URL : https://journals.openedition.org/hel/1422

DOI : 10.4000/hel. 1422

ISSN : 1638-1580

Éditeur

Société d'histoire et d'épistémologie des sciences du langage

\section{Édition imprimée}

Date de publication : 31 décembre 2021

Pagination : 201-205

ISBN : 9791091587150

ISSN : 0750-8069

Référence électronique

Julien Sibileau, «Larcher, Pierre. 2020. Sur le Coran. Nouvelles approches linguistiques », Histoire Épistémologie Langage [En ligne], 43-2 | 2021, mis en ligne le 01 décembre 2021, consulté le 17 décembre 2021. URL : http://journals.openedition.org/hel/1422 ; DOI : https://doi.org/10.4000/hel. 1422

Ce document a été généré automatiquement le 17 décembre 2021.

\section{c) (i) $\Theta$}

HEL is licensed under a Creative Commons Attribution-NonCommercial-NoDerivatives 4.0 International License 


\section{Larcher, Pierre. 2020. Sur le Coran. Nouvelles approches linguistiques}

Limoges : Lambert-Lucas. 235 p.

Julien Sibileau

\section{RÉFÉRENCE}

Larcher, Pierre. 2020. Sur le Coran. Nouvelles approches linguistiques. Limoges : Lambert-

Lucas. 235 p. ISBN : 978-2-35935-313-6.

1 Le linguiste qui entreprend d'étudier la langue du Coran constatera sans trop de difficultés que les publications académiques adoptant une approche linguistique du texte coranique sont rares. Si la codicologie, quant à elle, a développé une approche essentiellement axée autour de la matérialité du texte coranique, prenant pour objet les supports sur lesquels ont été inscrits des fragments de ce même texte, mais aussi les variations graphiques attestées dans ces fragments, il reste qu'elle n'a point cherché à dégager les problématiques linguistiques posées par le contenu graphique de ces fragments, encore moins par le contenu des deux lectures canoniques les plus répandues aujourd'hui dans le monde musulman : celle de Hafș 'an 'Așim (Coran du Caire), et celle de Warš 'an Näfi' (Coran du Maghreb). P. Larcher est sans nul doute l'un des seuls linguistes à avoir pris pour objet d'étude, non pas l'aspect matériel du texte coranique, ni l'histoire de son élaboration, tâche non moins importante entreprise par les codicologues, mais la langue du texte coranique. Dans Sur le Coran. Nouvelles approches linguistiques, il nous livre, grâce à son érudition, une des plus importantes contributions sur cette question, si ce n'est la plus importante à ce jour.

2 Composé de douze chapitres, répartis en cinq parties traversées par une thématique spécifique, ce volume interroge la langue du texte coranique selon différents points de vue. Le point de départ à partir duquel $\mathrm{P}$. Larcher construit son raisonnement est le texte, auquel il consacre la première partie de son ouvrage. "Qui dit texte, dit écrit ", note-t-il dès les premières lignes, en ajoutant que le système graphique dans lequel ce 
texte est écrit est "archaïque ", " défectif ", c'est-à-dire qu'il ne correspond pas aux règles orthographiques de l'arabe, que ce soit dans sa variété classique, ou dans sa variété moderne. Dès lors se pose la question de savoir ce que peut nous révéler la graphie du texte coranique du point de vue linguistique. À partir d'un constat voulant que ce qui est dit est tantôt écrit, tantôt non, P. Larcher nous invite à interroger le rapport entre le dit et l'écrit dans le Coran afin d'en proposer quelques interprétations. C'est là l'objet du chapitre I intitulé « Le Coran : le dit et l'écrit ». Prenant l'exemple de rasūlā (envoyé, messager) et sabila (chemin) en Coran 33:66 et 67, celui-ci note que les règles de l'orthoépie ne sont pas respectées puisqu'en contexte pausal, ces deux mots auraient dû être prononcés respectivement rasūl et sabïl. Pourquoi donc avoir ici inscrit le 'alif, marque de l'allongement vocalique $\bar{a}$, alors même que sabill, à l'accusatif, soit sabill(a), est noté sans 'alif au verset 4 de la même sourate, en dépit du même contexte prosodique (mots se terminant en -an et réalisés $\bar{a}$ en contexte pausal) ? L'analyse de cet exemple pose donc une question : faut-il suivre le rasm au risque de contrevenir au principe de la rime, bien mis en évidence par la graphie dans le reste de la sourate 33, ou bien faut-il respecter le principe de la rime malgré le constat de cette dissonance entre graphie et phonie? À travers l'étude d'autres exemples, P. Larcher met en évidence des distorsions entre le dit et l'écrit, une hétérogénéité qui, bien que reconnue par la tradition, n'en demeure pas moins sujette à des interprétations parfois erronées devant être écartées afin de proposer une histoire objective de la constitution du texte coranique.

3 Dans les deux premiers articles constitutifs de ce recueil, P. Larcher part donc d'un constat empirique qui est que le texte coranique canonique, dans sa variante du Caire et du Maghreb, atteste de nombreuses variations. Un certain nombre d'entre elles sont étudiées afin de contribuer à l'établissement d'une histoire du texte coranique, histoire dont la variation est à l'origine. Or s'interroger sur les variations attestées dans le texte coranique conduit nécessairement à poser la question de la langue du Coran. Le lecteur est donc amené à passer d'une approche principalement philologique à une approche linguistique historique.

4 La langue du Coran dans son contexte historique, tel est l'objet de la seconde partie de l'ouvrage, qui débute par le chapitre III intitulé « Arabe préislamique, arabe coranique, arabe classique : un continuum?». Dans cet article, P. Larcher cherche à établir une typologie des différents types d'arabe attestés dans l'Antiquité tardive, c'est-à-dire à partir des premières inscriptions en arabe épigraphique préislamique jusqu'à l'avènement de l'arabe dit "classique». Le premier type d'arabe est l'arabe épigraphique préislamique composé des langues nordarabiques, des inscriptions arabes empreintes d'araméismes au nord et de sabéismes au sud, ainsi que des inscriptions arabes rédigées dans un système graphique arabe. Vient ensuite l'arabe coranique qui est quant à lui attesté dès la fin du VII siècle à l'époque du calife omeyyade 'Abd alMālik (r. 65-86/685-705). Les premiers manuscrits en écriture coufique apparaissent au milieu $\mathrm{du} \mathrm{II}^{\mathrm{e}} / \mathrm{VIII}^{\mathrm{e}}$ siècle. Quant aux manuscrits en écriture hiğăzi ou $m \bar{a}^{\prime} i l$, ils sont paléographiquement datables de la seconde moitié du $\mathrm{e}^{\mathrm{er}} / \mathrm{VII}^{\mathrm{e}}$ siècle. P. Larcher pointe du doigt le fait qu'à la différence du matériel épigraphique de l'arabe préislamique, l'historien de la langue fait face à une pluralité de variantes: les qirā'āt, outre celle de Warš pour le Maghreb et Ḥafș pour Le Caire. Ce n'est que tardivement, au $\mathrm{x}^{\mathrm{e}}$ siècle, que ces «lectures " seront fixées au nombre de sept. Quant à l'étiquette "classique " pour désigner l'arabe des grammaires, P. Larcher met en évidence le fait que celle-ci ne 
renvoie pas à une variété d'arabe, mais plutôt qu'elle constitue une dénomination sociolinguistique. Cette vue est corroborée par le nom donné à l'arabe classique, qui témoigne d'une situation de pluralité, al-lugia l-fușhā, réécriture d'une expression plus ancienne : afșah al-lugāat al-'arabiyya (à peu près « la plus châtiée des manières de parler arabe $»$ ).

Le chapitre IV intitulé "Qu'est-ce que l'arabe du Coran? Réflexions d'un linguiste» s'inscrit donc dans la continuité du chapitre III. Dans celui-ci, P. Larcher opère un focus sur l'arabe coranique et rappelle que, s'agissant de la constitution du texte coranique, les islamologues travaillent à partir de trois hypothèses : (1) celle de Burton (1977) veut qu'un Coran écrit existe dès l'époque de Mahomet à Médine ; (2) pour Wansbrough (1977), la constitution du corpus coranique aurait au contraire été un travail de longue haleine s'étendant sur trois siècles; (3) pour la plupart des islamologues, le muṣhaf 'Uțmān serait le nom "conventionnel» donné à la version officielle imposée par le calife omeyyade 'Abd al-Mālik (r. 65-86/685-705). Reprenant un certain nombre d'exemples mentionnés dans les articles précédents, P. Larcher plaide en faveur d'une distinction entre arabe du Coran et arabe classique compte tenu du fait que l'arabe coranique présente des traits phonologiques, morphologiques, syntaxiques, lexicaux, orthographiques, qui ne seront pas retenus par l'arabe classique, variété de prestige et norme scolaire.

6 Dans le chapitre V intitulé «La langue du Coran: quelle influence sur la grammaire arabe? » l'auteur s'interroge sur le rapport entre la constitution du texte coranique et le développement de la grammaire arabe d'un point de vue linguistique. Selon lui, ce n'est pas la langue du Coran qui aurait déterminé le sort et le devenir de la grammaire arabe mais bien l'activité grammaticale, étalée dans le temps, qui aurait peu à peu classicisé la langue du Coran. Empreinte de multiples variantes, de traits préclassiques ou non classiques, cette langue, loin d'être un ensemble homogène, aurait subi le sort que bon nombre de langues ont connu dans leur histoire, à savoir une rétroprojection classicisante a posteriori. On pourra à ce titre effectuer un parallèle avec l'ouvrage de R. Anthony Lodge, French: From Dialect to Standard, dans lequel une approche sociolinguistique du développement de la langue française est proposée et où l'auteur rappelle que le français est un dialecte qui a eu plus de succès que les autres et qui a ensuite été standardisé.

7 La troisième partie aborde la question de la langue du Coran du point de vue du lexique. L'idée principale défendue dans le chapitre VI intitulé «Le concept de langue dans le Coran " est celle de la thèse vernaculaire de la langue du Coran, thèse notamment appuyée par deux versets dans lesquels est attestée l'expression bi-lisānika, « dans ta langue », id est, « dans ta langue arabe » (Coran 19:97 et 44:58) ainsi que le verbe yassara, glosé par le polygraphe égyptien al-Suyūṭī (m. 911/1505) en sahhala (faciliter) qui indique l'idée de faciliter la compréhension du message à ses destinataires, ce qui exigeait l'emploi d'un langage clair. P. Larcher met donc en avant la façon dont la langue du Coran a été mythifiée, acquérant au fil du temps un statut de prestige, alors même qu'elle ne semble au départ n'être qu'une langue vernaculaire hétérogène mêlant des traits linguistiques appartenant tant à l'arabe de l'ouest qu'à celui de l'est. Les deux chapitres suivants, respectivement intitulés «Le concept de paix et ses expressions en arabe » et « Ğihād et salām : guerre et paix en Islam ou le point de vue du linguiste ", pointent du doigt les dissonances entre interprétation moderne de certains termes coraniques et signification réelle de ces derniers remis dans leur contexte 
historique et sémitique. Il est ainsi montré que la racine $s-l-m$ (de laquelle dérivent les mots salām et islām) porte en elle la notion de paix par soumission, de même que le ğihād spirituel serait une réinterprétation métaphorique tardive dont on ne retrouve pas la trace dans le Coran. Outre le fait que cet article apporte au lecteur des informations à propos du concept de paix en arabe classique, on se rend surtout compte que $\mathrm{P}$. Larcher cherche à pallier l'absence de comparatisme linguistique dont pâtit la culture arabe classique, un silence contrastant avec la foisonnante érudition attestée dans les textes médiévaux de la culture hébraïque. De fait, celui-ci amène le lecteur à se demander pourquoi la culture arabe classique n'a point vu naître d'Ibn Qurayš et de Saadia Gaon qui - comme le signale à juste titre la regrettée Sophie Kessler-Mesguich, en citant G. Vajda (1954) - peuvent être considérés comme les pionniers de la grammaire comparée des langues sémitiques? Quelle que soit la réponse à cette question, qui nécessiterait certainement la tenue de colloques et de séminaires ad hoc, un esprit alerte ne manquera pas de faire le lien avec le qualificatif 'a'ğam (non arabe, étranger) qui, attesté en Coran 16:103, ne laisse place à aucun doute sur le fait que du vivant du prophète de l'Islam, le fait qu'un homme à la langue non arabe ('a'ğamī) puisse enseigner au prophète le contenu du Coran était une idée répandue. Bien que la trace de cette polémique puisse expliquer l'absence d'ouvrages à visée comparatiste dans la culture arabe classique, P. Larcher montre que la confrontation de certains éléments du lexique coranique avec d'autres tirés du matériel sémitique s'avère fructueuse et éclairante.

8 La quatrième partie, consacrée au discours, débute par un article (chapitre IX) intitulé "Coran et théorie linguistique de l'énonciation», dans lequel P. Larcher prédit que l'étude des qiră'āt connaitra bientôt un retour en grâce notamment par le biais de l'histoire de la grammaire arabe. Pour ce qui est de la langue du texte coranique, l'hypothèse de la koinè ne l'emporte pas, de l'avis de P. Larcher, sur l'hypothèse de Vollers, à savoir celle d'une homogénéisation a posteriori de l'arabe poético-coranique. Même la langue du matériel épigraphique ne saurait nous éclairer sur ce qu'est l'arabe classique, compte tenu de la paucité des données recueillies par les épigraphistes. Quoi qu'il en soit, P. Larcher rappelle que l'arabe coranique a permis à un certain nombre de savoirs médiévaux de développer une méthodologie autour d'un objet: le kalām ou le Hițāb, le premier pouvant désigner la langue ou le discours, alors que le second ne désigne que le discours. C'est notamment le cas des uṣūl al-figh (principes de la jurisprudence) où le Hitâab occupe une place centrale. Pour P. Larcher, le mécanisme d'interprétation juridique du Coran n'est autre qu'une théorie de l'énonciation, en particulier des actes illocutionnaires énoncés par Allāh et reçus par le destinataire (mukallaf). Le second article de cette partie, intitulé « Négation et rectification en arabe coranique : la structure $m \bar{a} f a^{\prime} a l \bar{a} . .$. wa-lākin... ", étudie la corrélation entre connecteurs rectificatifs et types de négation à partir de l'analyse du connecteur lākin. À travers cette étude, P. Larcher propose de reformuler la description d'Anscombre et Ducrot: quand $p$ est négatif, cette négation est toujours modale avec SN (fonction réfutative à l'instar de bal), mais peut être aussi bien modale que descriptive avec PA (fonction argumentative à l'instar de lākin), ce qui permet de reconnaître les structures de type lam + inaccompli (négation modale) suivie de läkin comme en Coran 49:14. On voit très bien, à travers cet exemple, comment l'étude de la langue du Coran permet aussi de contribuer au développement même de la linguistique. 
9 La cinquième et dernière partie est consacrée à la Koranphilologie médiévale, nom que les orientalistes allemands ont attribué aux différentes réflexions produites par les grammairiens sur le texte coranique. Le premier article de cette partie s'intitule « Les $M a^{\prime} a \bar{n} \bar{i}$ al-Qur'ān d'al-Farrā' ou la théologie tempérée par la philologie ». Dans celui-ci, P. Larcher met en avant la manière dont l'activité grammaticale médiévale a en quelque sorte cherché à «classiciser » l'arabe du Coran à partir de l'exemple bien connu de Coran 20:63 dans lequel le démonstratif du duel hādāni gouverné par la particule inna (ductus alif, $n, h, d, n$ ) n'est pas à l'accusatif. Rappelant que la tradition classique évoque ici une erreur (lahnn) à travers la voix du grammairien de Kūfa al-Farrāà (m. 207/822), P. Larcher met en évidence le décalage entre le réalisme des classiques et l'ignorance d'une certaine apologétique moderne, et retrace les multiples pistes qui ont été explorées dans les textes anciens afin d'attribuer une explication à ce fait, jusqu'à celle de l'erreur commise par un copiste, nous ramenant ainsi à la matérialité du texte coranique. Enfin, le second article de cette partie, et dernier de l'ouvrage, intitulé "Neuf traditions sur la langue coranique rapportées par al-Farrā' et alii ", prolonge la discussion entamée dans l'article précédent. Dans celui-ci, P. Larcher analyse un texte attribué à al-Farrā', édité par Paul Kahle en 1947, et intitulé 'adad āy al-Qur'ān (Sur le nombre des versets du Coran). La première partie de ce texte viserait à concilier la thèse théologique consistant à dire que la langue du Coran est al-luǵa al-fuṣhă, soutenue notamment au $x^{e}$ siècle par le lexicographe Ibn Fāris (m.395/ 1004), et celle des philologues pour qui cette langue était celle de bédouins. Quant à la seconde, elle contient un récit attribué à Ibn Mas'ūd (m. 32/650) laissant à penser que le Coran aurait pu être récité sans la flexion casuelle, et qu'au demeurant celle-ci pourrait être rattachée à une fonction mélodique, non syntaxique.

Pour conclure, nous pouvons dire que l'approche historique de la langue du Coran adoptée par P. Larcher dans cet ouvrage fondateur semble faire écho à «la révolution qu'ont connue au XIX ${ }^{e}$ siècle en Occident les études bibliques, avec la méthode historicocritique, fondée sur la philologie » (p. 188). Cette approche nous amène à réfléchir de manière générale sur la relation entre l'établissement d'un corpus sacré et la création d'une norme linguistique à travers une langue littérarisée. On ne manquera pas de constater, par exemple, quelques similitudes entre l'entreprise de traduction de la Bible par Luther en Allemagne au $\mathrm{xvI}^{\mathrm{e}}$ siècle, et l'idée de révélation en langue claire (Coran 26:195), en ce que Luther, dans ses Écrits sur la traduction, qualifie l'allemand dans lequel il a traduit la Bible et le Nouveau Testament de « bon et clair », en allemand "rein und klar deutsch» (p. 84-85). Dans les deux cultures, arabe musulmane et allemande protestante, l'irruption d'un texte sacré en langue vernaculaire s'accompagne d'une réflexion linguistique d'ampleur, fondatrice, et concomitante avec le premier. La réflexion menée par P. Larcher dans les différents articles constitutifs de cet ouvrage offre au lecteur des pistes de lecture plus générales ouvrant donc la voie au comparatisme interculturel. L'approche plurielle du texte coranique proposée ici constitue une contribution considérable à son étude linguistique et ne manquera pas d'inciter les chercheurs à suivre cette voie en essayant de redonner aux sources anciennes en langue arabe une certaine fraîcheur. 


\section{BIBLIOGRAPHIE}

Déroche, François. 2019. Le Coran, une histoire plurielle. Paris : Seuil.

Kahle, Paul. 1947, 1959. The Cairo Geniza. Oxford : Basil Blackwell.

Lodge, R. Anthony. 1993. French: From Dialect to Standard. Londres : Routledge.

Luther, Martin. 2017. Écrits sur la traduction. Paris : Les Belles Lettres.

\section{AUTEURS}

\section{JULIEN SIBILEAU}

Inalco, Centre de recherches Moyen-Orient Méditerranée (Cermom) 